HUTP-98/A001

PUPT-1758

UCSD/PTH 98-04

\title{
Misleading Anomaly Matchings?
}

\author{
John Brodie ${ }^{1}$, Peter Cho $^{2}$ and Kenneth Intriligator ${ }^{3}$ \\ ${ }^{1}$ Department of Physics, Princeton University, Princeton, NJ 08540 \\ ${ }^{2}$ Lyman Laboratory, Harvard University, Cambridge, MA 02138 \\ ${ }^{3}$ Department of Physics, University of California at San Diego, \\ 9500 Gilman Drive, La Jolla, CA 92093
}

\begin{abstract}
We investigate the low energy dynamics of $\mathcal{N}=1$ supersymmetric $S O(N)$ gauge theories with a single symmetric tensor matter field. These theories exhibit non-trivial matching of global 't Hooft anomalies at the origin of moduli space. We argue that their quantum moduli spaces possess distinct Higgs and confining branches which touch at the origin in an interacting non-Abelian Coulomb phase. The matching of anomalies between microscopic degrees of freedom and colorless moduli therefore appears to be coincidental. We discuss a formal mathematical relation between the $S O(N)$ model and an analogous $S p(2 N)$ theory with a single antisymmetric matter field which provides an explanation for the anomaly matching coincidence.
\end{abstract}


Recent advances in analyzing the strong coupling dynamics of supersymmetric gauge theories have opened up several new directions for model building [1]. One interesting application has been to the study of dynamical supersymmetry breaking. A number of theories utilizing novel dynamical mechanisms to break SUSY have been constructed during the past few years [2]. One especially simple proposal is based upon an $S U(2)$ model with a single isospin-3/2 matter field [3]. Nontrivial 't Hooft anomaly matching in this chiral model suggests that it confines with a smooth quantum moduli space. Supersymmetry breaking then results upon adding a tree level superpotential. However, there is another possibility for the $S U(2)$ model's low energy dynamics [3]: the 't Hooft anomaly matching may be coincidental and the theory may have a non-trivial RG fixed point at the origin. In this case, supersymmetry need not be broken upon adding the tree level superpotential. Which scenario is correct remains an unsettled question.

In this note, we examine a simple class of $\mathcal{N}=1$ supersymmetric $S O(N)$ models with a single matter chiral superfield $S$ in the two-index, symmetric, traceless, tensor representation $\square$. As we shall see, these models are similar to the $S U(2)$ theory inasmuch as they exhibit non-trivial 't Hooft anomaly matching, which suggests confinement with a smooth moduli space. 1 We will argue, however, that the moduli space for these theories must have a more intricate structure, with various branches and a non-trivial RG fixed point at the origin. The anomaly matching then appears to be coincidental. A skeptic might view the $S O(N)$ models as casting doubt on the proposed confinement and supersymmetry breaking of the model of [3]. At the very least, they demonstrate that anomaly matching can be misleading.

It is important to recall that the $S$ field does not transform according to a faithful representation of the $S O(N)$ gauge group's center. Test charges in spinor or vector representations cannot be screened by either gluons or dynamical $S$ matter fields. Therefore, the $S O(N)$ model's moduli space can à priori have distinct Higgs, confining and oblique confining branches where Wilson and 't Hooft loops exhibit various possible area and perimeter law scalings. This feature of the $S O(N)$ model represents a clear qualitative difference with the $S U(2)$ theory of [3] whose $I=3 / 2$ field does provide a faithful representation of the $Z_{2}$ center of $S U(2)$.

1 These models were recently considered in [- 4 as part of a complete classification of all theories based on simple gauge groups with a freely generated moduli space and matching 't Hooft anomalies. Some of our previously unpublished observations on these models were cited in that work. The $S O(N)$ theories were also recently constructed via branes in [5]. 
In the absence of any tree level superpotential, the $S O(N)$ model has an anomaly free $U(1)_{R}$ symmetry with $R(S)=4 /(N+2)$. 2 Its one loop beta function is $b_{0}=2(N-4)$, so the $N \geq 5$ theories are asymptotically free. Although $b_{0}=0$ for the $S O(4) \cong S U(2) \times$ $S U(2)$ model with $S \sim(\mathbf{3}, \mathbf{3})$, this theory is not asymptotically free at two loop order. It thus flows to a free theory in the IR. Similarly, the $S O(2)$ and $S O(3)$ theories are not asymptotically free and flow to free theories in the IR.

The $S O(N)$ model possesses a moduli space of classical vacua given by solutions to the $D$-flatness condition modulo gauge transformations. $D_{a}=\operatorname{Tr}\left(T_{a} S S^{\dagger}\right)=0$ implies that the real and imaginary parts of $S$ commute and can be simultaneously diagonalized by an $S O(N)$ rotation. The moduli space is consequently $N-1$ complex dimensional. Throughout its bulk, the gauge group is generically completely broken by the Higgs mechanism. But there exist subspaces of enhanced gauge symmetry where some diagonal expectation values of $S$ are equal. On the subspace where

$$
\langle S\rangle=\left(\begin{array}{cccc}
z_{1} \mathbf{1}_{m_{1} \times m_{1}} & & & \\
& z_{2} \mathbf{1}_{m_{2} \times m_{2}} & & \\
& & \ddots & \\
& & & z_{\ell} \mathbf{1}_{m_{\ell} \times m_{\ell}}
\end{array}\right)
$$

with $\sum_{i=1}^{\ell} m_{i}=N$ and $\sum_{i=1}^{\ell} m_{i} z_{i}=0$, the low energy theory reduces to $l$ decoupled $S O\left(m_{i}\right)$ models, each with a traceless two index symmetric tensor $\square$, and $l-1$ singlet moduli corresponding to the $z_{i}$.

The $N-1$ dimensional classical moduli space is freely generated by arbitrary expectation values of the gauge invariant operators

$$
O_{n}=\operatorname{Tr} S^{n}, \quad n=2,3, \cdots, N
$$

One can also form the additional composite $B=\operatorname{det} S$. But it is linearly related by the trace of the characteristic polynomial for matrix $S$

$$
O_{N}-\frac{1}{2} O_{2} O_{N-2}-\frac{1}{3} O_{3} O_{N-3}+\cdots+(-1)^{N} N B=0
$$

to the operators in (2). This last expression can be used to eliminate $B$ regardless of any possible quantum corrections.

2 We adopt the $S O(N)$ index values $\mu(\square)=2, \mu(\operatorname{Adj}=$ 日) $=2 N-4$ and $\mu(\square)=2 N+4$ which count numbers of fermion zero modes in a single instanton background. 
In the quantum theory, any dynamically generated superpotential which could lift the classical moduli space degeneracy is determined by holomorphy and symmetry considerations to be of the form

$$
W_{\mathrm{dyn}}=C\left[\frac{S^{2 N+4}}{\Lambda^{2 N-8}}\right]^{\frac{1}{4}}
$$

where $\Lambda$ denotes the $S O(N)$ scale and $S^{2 N+4}$ stands for some function of the $O_{n}$ operators which has $S$ number equal to $2 N+4$. Asymptotic freedom requires the classical moduli space to be recovered in the weak coupling $\langle S\rangle / \Lambda \rightarrow \infty$ limit. This condition is incompatible with the form of $W_{\mathrm{dyn}}$ which yields a potential that grows with $S$. Consequently, the constant $C$ must vanish. We will refer to this part of the moduli space where $W_{\mathrm{tot}}=W_{\mathrm{dyn}}+W_{\text {tree }}=0$ as the "Higgs branch." Shortly, we will argue that the $W_{\mathrm{dyn}}$ in (4) with $C \neq 0$ must be generated on another "confining branch" of the theory when $W_{\text {tree }} \neq 0$.

Although strong dynamics do not generate any superpotential on the Higgs branch, they can still alter the theory's vacuum structure and lead to interesting phenomena near the origin. Classically, the moduli space metric for the $O_{n}$ fields has singularities on subspaces of the vacuum manifold where the gauge group is not completely broken. In the classical theory, massless $\square i$ matter fields and $S O\left(m_{i}\right)$ gluons must be included in order to obtain a non-singular description. In the quantum theory, the moduli space singularities are either smoothed out or else reflect possibly different massless fields.

Since the global $U(1)_{R}$ symmetry remains unbroken at the origin, it is possible to check 't Hooft anomalies in order to constrain the massless spectrum at this point. In the microscopic theory, the $U(1)_{R}$ and $U(1)_{R}^{3}$ anomalies assume the values

$$
\begin{aligned}
& A_{U(1)_{R}}=\left[\left(\begin{array}{c}
N+1 \\
2
\end{array}\right)-1\right]\left[-\frac{N-2}{N+2}\right]+\left(\begin{array}{c}
N \\
2
\end{array}\right)[1]=N-1 \\
& A_{U(1)_{R}^{3}}=\left[\left(\begin{array}{c}
N+1 \\
2
\end{array}\right)-1\right]\left[-\frac{N-2}{N+2}\right]^{3}+\left(\begin{array}{c}
N \\
2
\end{array}\right)[1]^{3}=\frac{(N-1)\left(5 N^{2}-4 N+4\right)}{(N+2)^{2}} .
\end{aligned}
$$

In the effective theory, we find the following contributions from the fermionic components of the $O_{n}$ moduli superfields:

$$
\begin{aligned}
& A_{U(1)_{R}}=\sum_{n=2}^{N}\left[\frac{4 n}{N+2}-1\right]=N-1 \\
& A_{U(1)_{R}^{3}}=\sum_{n=2}^{N}\left[\frac{4 n}{N+2}-1\right]^{3}=\frac{(N-1)\left(5 N^{2}-4 N+4\right)}{(N+2)^{2}} .
\end{aligned}
$$


The anomalies precisely match! This nontrivial agreement suggests that the $O_{n}$ composites saturate the massless spectrum of the quantum theory. If so, the quantum Kahler metric for the $O_{n}$ moduli should be flat near the origin and non-singular throughout the moduli space. This anomaly matching represents circumstantial evidence for confinement in the $S O(N)$ model in the same way as for the $S U(2)$ model of [3].

It is interesting to further consider discrete anomalies [6] 8]. Global anomalies for $Z_{N}$ groups should match between high and low energy descriptions of any gauge theory [9]. In

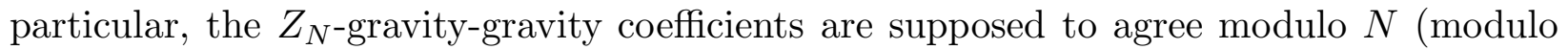
$N / 2$ ) for $N$ odd (even). On the other hand, other anomalies such as $Z_{N}^{3}$ and $Z_{N}^{2} U(1)_{R}$ can be corrupted by unknown massive state contributions. Therefore, they cannot be used to definitively rule out a proposed massless confining phase spectrum.

In the $S O(N)$ model, instantons break the classical $S$-number symmetry down to a $Z_{2 N+4}$ subgroup [10]. 3 After assigning the $S$ field charge 1 under this discrete group, we find that the $Z_{2 N+4}^{3}, Z_{2 N+4}^{2} U(1)_{R}$ and $Z_{2 N+4} U(1)_{R}^{2}$ anomalies do not match for general $N$. However, the $Z_{2 N+4}$ anomalies calculated in the microscopic gauge theory and low energy sigma model are equal:

$$
A_{Z_{2 N+4}}=\left[\frac{N(N+1)}{2}-1\right](1)=\sum_{n=2}^{N} n=\frac{(N-1)(N-2)}{2} .
$$

These discrete anomaly matching results are therefore consistent with the hypothesis that the $S O(N)$ model confines and yields a free field theory at the origin.

Although the picture of simple confinement at the origin passes all non-trivial anomaly tests, it can not provide a complete description of the moduli space. The first problem arises along subspaces of enhanced gauge symmetry within the Higgs branch where $S O(N) \rightarrow$ $\prod_{i} S O\left(m_{i}\right)$ with some $m_{i}=2,3$ or 4 . On these subspaces, the low energy theory is not asymptotically free. The massless spectrum must therefore include $S O\left(m_{i}\right)$ gauge fields and $\square \square_{i}$ matter fields. Since these subspaces intersect the origin, the massless spectrum at $\langle S\rangle=0$ can not simply consist of the confined $O_{n}$ moduli. We note that no such freeelectric subspaces enter into the $S U(2)$ model of [3] or other theories which are believed to confine.

Another way in which there could exist a free theory at the origin would be to have the same massless spectrum at $\langle S\rangle=0$ as that which exists on the free-electric subspace with

3 A $Z_{(N+2) / 4}$ subgroup of $Z_{2 N+4}$ is contained within $U(1)_{R}$. But there is no loss in working with the larger $Z_{2 N+4}$ symmetry since $U(1)_{R}$ anomalies are already known to match. 
maximal unbroken gauge group. For example when $N$ is a multiple of four, the massless spectrum on the free-electric subspace with maximal unbroken gauge group consists of free-electric $S O(4)^{N / 4}$ gauge fields and symmetric tensors, along with a subset of the $O_{n}$ operators which parameterize this subspace. This massless spectrum could conceivably extend down to the origin of moduli space. However, there seems to be no way to make this scenario compatible with the required 't Hooft anomaly matching at the origin. It thus appears that the theory at the origin can not be free and that the 't Hooft matching observed above is simply a misleading coincidence.

Another reason why confined $O_{n}$ fields cannot represent the complete massless spectrum at the origin of moduli space may be seen by turning on a tree level superpotential

$$
W_{\text {tree }}=\sum_{n=2}^{N} g_{n} O_{n} .
$$

In the presence of these classical terms, the eigenvalues $z$ of $S$ represent different solutions of $\sum_{n=1}^{N} n g_{n} z^{n-1}=0$. 6 Various vacua exist where different numbers of eigenvalues are equal. The general low energy theory is $\mathcal{N}=1$ pure Yang-Mills with a $\prod_{i} S O\left(m_{i}\right)$ gauge group. For $m_{i} \geq 3, S O\left(m_{i}\right)$ confines and yields $m_{i}-2$ supersymmetric vacua when $m_{i} \geq 5$, four vacua when $m_{i}=4$ and two vacua when $m_{i}=3$. The low energy spectrum contains a photon for each $m_{i}=2$.

For simplicity, consider the special case where only the mass coupling $g_{2}=\frac{1}{2} m$ in (8) is nonzero. For $m \gg \Lambda$, the heavy $S$ can be integrated out. The resulting low energy $S O(N)$ Yang-Mills theory is known to confine with a mass gap and to have $N-2$ supersymmetric vacua. If the original $S O(N)$ model simply had a moduli space with confinement in terms of the $O_{n}$ fields, turning on $W_{\text {tree }}=\frac{1}{2} \mathrm{mO}_{2}$ would lead to dynamical SUSY breaking in a similar fashion to the proposed mechanism in [3]. This would contradict the fact that the low energy theory has $N-2$ supersymmetric vacua.

Gluino condensation in the low energy $S O(N)$ Yang-Mills theory generates the superpotential

$$
W_{\mathrm{lo}}=\frac{1}{2}(N-2)\left[16 \Lambda_{\mathrm{lo}}^{3(N-2)}\right]^{1 /(N-2)}
$$

where the low energy scale is fixed by the matching relation $\Lambda_{\mathrm{lo}}^{3(N-2)}=m^{N+2} \Lambda^{2(N-4)}$. This result can be recovered from the effective superpotential

$$
W_{\text {conf }}=-\left[\frac{O_{2}^{N+2}}{(N+2)^{N+2} \Lambda^{2 N-8}}\right]^{1 / 4}
$$

4 The coupling $g_{1}$ is a Lagrange multiplier which enforces the tracelessness condition for $S$. 
in the upstairs theory after adding $W_{\text {tree }}=\frac{1}{2} m O_{2}$ and integrating out $O_{2}$. This superpotential is of the form (田). Since we have already argued that it is absent on the Higgs branch, we conclude that there must exist another "confining branch" of the theory on which $W_{\text {conf }}$ is generated when $m \neq 0$. 5

The multi-branch structure of the $S O(N)$ model's moduli space is reminiscent of that for $S U(2)$ with two adjoints [11,12]. We sketch its basic features in fig. 1. The plane in the figure represents the Higgs branch which is strongly coupled inside the shaded region nearby the origin and weakly coupled in the domain far away from $\left\langle O_{n}\right\rangle=0$. Partial free electric subspaces within the Higgs branch which intersect the origin are illustrated by the diagonal line in fig. 1 . When $m \neq 0$, the Higgs branch is lifted, and the theory resides on the confining branch represented by the cone. For any fixed value of $m$, the vacuum on the confining branch consists of $N-2$ points with a mass gap. As a result, there are no massless moduli associated with the confining branch. As can be seen from the expectation value $\left\langle O_{2}\right\rangle=(N+2)\left(16 m^{4} \Lambda^{2 N-8}\right)^{1 /(N-2)}$, small values for $m$ yield vacua which lie within the strongly coupled region nearby the moduli space origin. The spreading of the cone with increasing $m$ mimics the behavior of $\left\langle\mathrm{O}_{2}\right\rangle$. Points located on the confining branch at $m \gg \Lambda$ are still strongly coupled, for the low energy limit of the $S O(N)$ model at such points is a super Yang-Mills theory. The entire cone is thus shaded grey.

In fig. 1, the Higgs and confining branches are shown touching at the origin much as in $S U(2)$ theory with two adjoints [11,12]. The origin must then reside in an interacting non-Abelian Coulomb phase, for no free field spectrum could incorporate both the Higgs and confining branches at this point.

It is also possible, though unlikely, that the distinct Higgs and confining branches for the $S O(N)$ model do not meet. Such a disconnected branch structure actually does occur in $S O\left(N_{c}\right)$ theory with $N_{f}=N_{c}-4$ vector flavors [11]. Two inequivalent branches arise from aligned and misaligned gaugino condensates within the low energy unbroken $S O(4) \cong S U(2) \times S U(2)$ gauge group. On the Higgs branch, $W_{\text {dyn }}$ vanishes as a result of cancellation between the two gaugino condensates, 't Hooft anomalies match at the origin and the low energy spectrum contains only the massless moduli. On the confining branch,

5 When other source terms besides $g_{2}=\frac{1}{2} m$ in the tree level superpotential in (8) are nonzero, more general confining phase superpotentials involving $O_{n>2}$ must be generated. The confining branch must also include the vacua with different unbroken $S O\left(m_{i}\right)$, including the ones with $m_{i}=2$ which have a massless photon. 


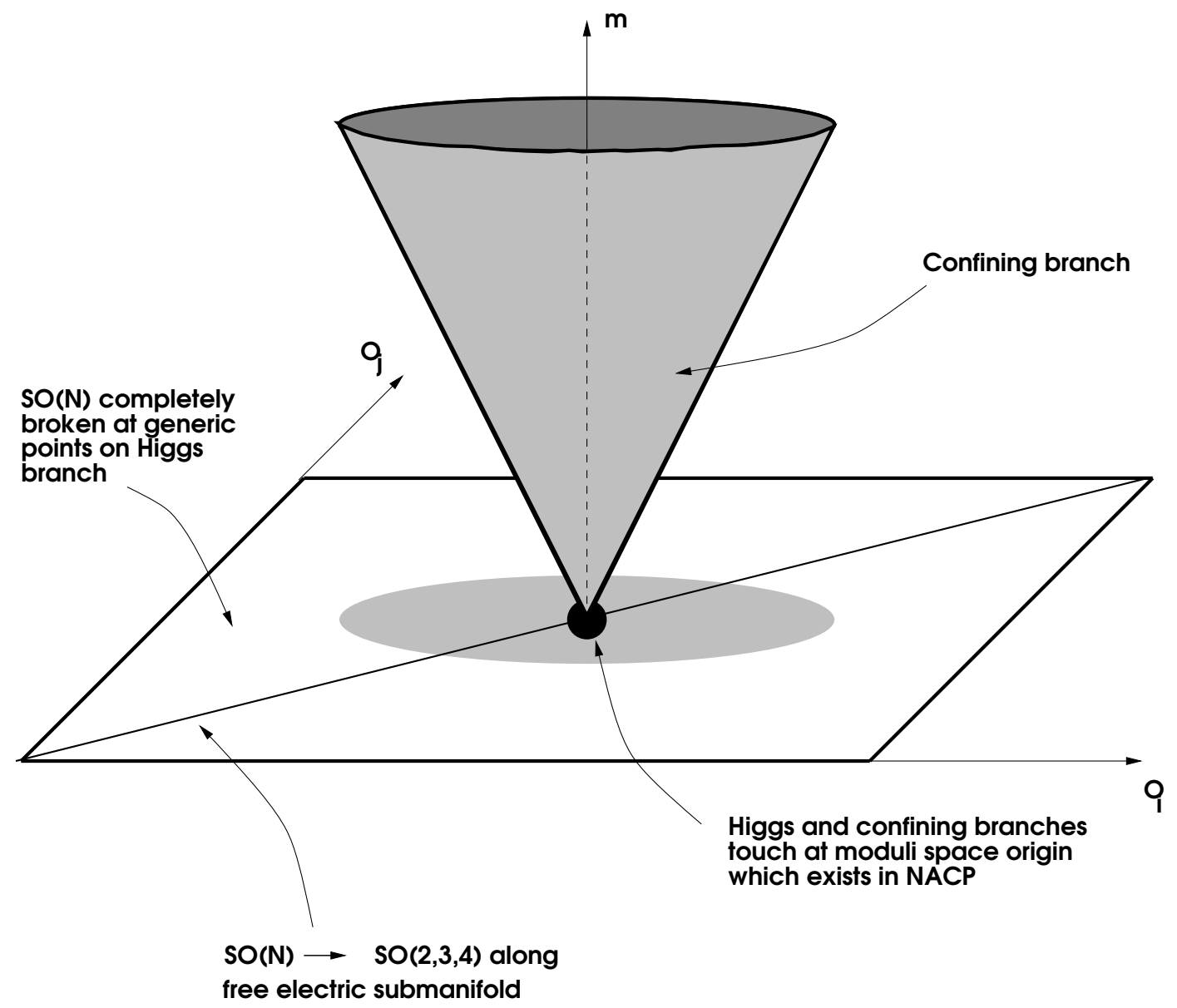

Fig. 1: A schematic picture of the $S O(N)$ model's quantum moduli space.

a destabilizing superpotential is dynamically generated. When $W_{\text {tree }} \neq 0$, the Higgs branch ceases to yield supersymmetric vacua and is eliminated, and the low energy theory lives on the confining branch. Since gaugino condensates do not appear at generic points within our $S O(N)$ model's moduli space where the gauge group is completely broken, we do not expect the theory with a symmetric matter field to exhibit such a disconnected branch structure.

More generally, we expect that the theories recently classified in [4] for which the gauge group can (can not) be completely broken do not (do) possess disconnected Higgs and confining branches associated with aligned and misaligned gaugino condensates in the unbroken product gauge group. The general distinction between the two cases may be seen by turning on a mass term $m$. On the confining branch, the expectation value of a matter field $Q$ with index $\mu$ is fixed by symmetries and holomorphy to be $\langle Q\rangle \sim$ $\left(m^{\mu-G} \Lambda^{3 G-\mu}\right)^{1 / 2 G}$, where $G$ denotes the adjoint index. If $\mu<G$, the gauge group generally breaks to a non-trivial subgroup and the moduli run off to infinity as $m \rightarrow 0$. The confining 
branch can then be thought of as a cone turned upside-down relative to that in fig. 1 . The cone approaches, but never touches, the plane at infinite moduli values. The Higgs and confining branches are therefore disconnected when $\mu<G$. In contrast, it seems more likely that the Higgs and confining branches intersect at the origin in a non-Abelian Coulomb phase for $\mu>G$ theories where the gauge group can be completely broken. The $S O(N)$ model with a single symmetric matter field falls into the latter category.

The structure of the theory at the origin can be probed by perturbing the $S O(N)$ model with $W_{\text {tree }}=\lambda \operatorname{Tr} S^{k+1}$ for $k \geq 2$. The equations of motion for the eigenvalues of $S$ are $\lambda(k+1) z^{k}+g_{1}=0$, where $g_{1}$ is a Lagrange multiplier that enforces the tracelessness condition. These equations generally only have the trivial solution $z=g_{1}=0$. The superpotential thus lifts the moduli space. When $N=m k$ with $m$ an integer, there is a one complex dimensional moduli space of vacua which looks like $\langle S\rangle=z \operatorname{diag}\left(\mathrm{e}^{2 \pi \mathrm{i} \ell / \mathrm{k}}\right) \otimes 1_{\mathrm{m} \times \mathrm{m}}$ where $z \in \mathbf{C}$ and $\ell=1, \cdots, k$. This vev breaks $S O(N) \rightarrow S O(m)^{k}$, and all massless matter fields are eaten. For all values of $N$ and $k$, the theories with $W_{\text {tree }}=\lambda \operatorname{Tr} S^{k+1}$ can be regarded as the $N_{f}=0$ limit of a class of $S O(N)$ models analyzed in ref. [13] with a single symmetric matter field and $N_{f}$ vectors. It has a dual description in terms of an $S O(4 k-N)$ gauge group, a traceless symmetric tensor $\widetilde{S}$ and a tree level superpotential $\widetilde{W}_{\text {tree }}=\tilde{\lambda} \operatorname{Tr} \widetilde{S}^{k+1}$. This duality follows from the tower of dual pairs found in [13] for general $N_{f}$ after all vector matter fields are given masses and integrated out.

A special case occurs when $N=2 k$. $W_{\text {tree }}$ then respects the anomaly free $U(1)_{R}$ symmetry, and the electric and magnetic theories are identical. A one complex dimensional moduli space remains unlifted by $W_{\text {tree }}$ along which the gauge group is broken to $S O(2)^{k}$. The massless spectrum consequently contains $k$ photons. In the particular case of $S O(4) \cong$ $S U(2) \times S U(2)$ theory with $S \sim(\mathbf{3}, \mathbf{3})$ and $W_{\text {tree }}=\lambda S^{3}$, a two-dimensional manifold of non-trivial $\mathcal{N}=1$ renormalization group fixed points emanates out from the origin as a function of $g_{1}, g_{2}$, and $\lambda$ [14]. For $N=2 k>4$, there can not be an analogous non-trivial $\mathrm{RG}$ fixed point at the origin of the moduli space since the theories are asymptotically free with a dynamical scale $\Lambda$. However, there could be non-trivial RG fixed points for $N=2 k>4$ away from the origin, with a line of fixed points as a function of $\lambda$ and $\langle S\rangle / \Lambda$. This scenario requires the spontaneous breaking of scale invariance for $\langle S\rangle \neq 0$ and the explicit breaking due to $\Lambda$ to cancel. The duality of [13] would then provide a dual description of this fixed point.

For general $N$ and $k, W_{\text {tree }}\left(\widetilde{W}_{\text {tree }}\right)$ breaks $U(1)_{R}$ in the electric (magnetic) theories. In the far infrared, an accidental $U(1)_{R}$ symmetry must be restored as part of 
the super-conformal algebra of the (possibly free) IR fixed point. When the gauge coupling is strong, the appropriate $U(1)_{R}$ symmetry in the same super-multiplet as the stress tensor should be close to the anomaly free one. The statement that the superpotential generally violates this $U(1)_{R}$ symmetry is then equivalent to saying that it is generally relevant or irrelevant, rather than marginal. In cases where the dual $S O(4 k-N)$ gauge group is not asymptotically free and the magnetic superpotential also appears irrelevant $\left(D\left(\widetilde{W}_{\text {tree }}\right)=\frac{3}{2} R\left(\widetilde{W}_{\text {tree }}\right) \Longrightarrow 2 k \leq N\right)$, it seems likely that the dual becomes free in the infrared. The requirement that these dual or free magnetic theories be recovered when the original $S O(N)$ model is perturbed again favors the hypothesis that various moduli space branches meet at the origin in a non-Abelian Coulomb phase, which allows for nontrivial low energy dynamics.

To recapitulate, we have given three arguments for why the $S O(N)$ model's moduli space origin exists in an interacting non-Abelian Coulomb phase. Firstly, it is difficult to reconcile the 't Hooft anomaly matching results with the existence of free electric fields along subspaces that intersect the origin. Secondly, the moduli space must have a confining branch when the symmetric matter field is given a nonzero mass. This confining branch most likely touches the Higgs branch at the origin. Finally, a nontrivial phase and branch structure must arise when the original $S O(N)$ model is perturbed with a general tree level superpotential. Perhaps a dual description of the RG fixed point at the origin can be found which would provide a weak coupling picture for the confining branch and other phenomena associated with $W_{\text {tree }} \neq 0$. At present, no such dual is known.

In light of all these findings, we conclude that the $S O(N)$ model represents a rare example where global anomaly matching does not signal simple confinement. The existence of an infinite chain of theories where anomaly matching appears to be misleading represents a worthwhile point to bear in mind when analyzing the infrared behavior of other supersymmetric and nonsupersymmetric models.

If the $S O(N)$ theory does not confine, why do the anomalies match? Some insight into this coincidence can be gained from the negative dimensional group theory relationship

$$
S O(2 N)=\overline{S p(-2 N)}
$$

where the overbar indicates interchange of symmetrization and antisymmetrization [1520]. This formal expression links orthogonal groups acting on conventional bosonic tensor spaces to negative dimensional symplectic groups defined by their action on tensors in 
Grassmann vector spaces. For instance, the dimension of an $S O(2 N)$ irrep labeled by a Young tableau $\lambda$ can be obtained up to a sign from that for the corresponding $S p(2 N)$ irrep with the transposed tableau $\lambda^{T}$ by simply setting $N \rightarrow-N$. Similarly, every $S O(2 N)$ invariant scalar is related to an $S p(2 N)$ counterpart by replacing the symmetric $g_{\alpha \beta}$ metric with its antisymmetric $J_{\alpha \beta}$ analogue and swapping $N \rightarrow-N$.

The group theory relation in (11) suggests that the $S O(N)$ model is mathematically similar to the theory with symmetry group

$$
\widetilde{G}=S p(2 N)_{\text {local }} \times U(1)_{R}
$$

and matter content

$$
A \sim\left(日 ;-\frac{4}{2 N-2}\right)
$$

which was studied in refs. [21,22]. Like its orthogonal counterpart, this symplectic supersymmetric model possesses $N-1$ complex flat directions which are labeled by the operators

$$
\widetilde{O}_{n}=\operatorname{Tr}(A J)^{n}, \quad n=2,3, \cdots, N .
$$

At generic points in moduli space, the expectation value for $A$ breaks $S p(2 N) \rightarrow S U(2)^{N}$. The quantum moduli space consequently has a variety of disconnected branches which are associated with the different possible signs for the gaugino condensates in each of the $S U(2)$ factors of the unbroken gauge group. The condensate sum generally yields non-vanishing dynamical superpotentials, which lift the classical vacuum degeneracy. But on one branch, the different condensate contributions precisely cancel and $W_{\mathrm{dyn}}=0$. 6 The moduli space on this Higgs branch is smooth in terms of the $\widetilde{O}_{n}$ fields, and the 't Hooft anomalies

$$
\begin{aligned}
& A_{U(1)_{R}}=-2 N-1 \\
& A_{U(1)_{R}^{3}}=\frac{(-2 N-1)\left(5 N^{2}+2 N+1\right)}{(-N+1)^{2}}
\end{aligned}
$$

match at the microscopic and macroscopic levels. Comparing these anomaly expressions with their analogues in (6), we observe

$$
\begin{array}{lcc}
A_{U(1)_{R}}^{S p(2 N)} & \stackrel{2 N \rightarrow-N}{\longrightarrow} & A_{U(1)_{R}}^{S O(N)} \\
A_{U(1)_{R}^{3}}^{S p(2 N)} & \stackrel{2 N \rightarrow-N}{\longrightarrow} & A_{U(1)_{R}^{3}}^{S O(N)}
\end{array}
$$

6 After taking into account scale matching relations, one can check that there is a Higgs branch with such a cancellation for all $N$. 
So matching of the $U(1)_{R}$ and $U(1)_{R}^{3}$ anomalies in the $S O(N)$ theory with a single symmetric matter field appears to be an automatic mathematical consequence of the same anomaly matchings in the $S p(2 N)$ theory with an antisymmetric field. In the symplectic model, the matching is physically significant and signals genuine confinement.

Given the connection between the $S O$ and $S p$ anomalies, it is amusing to note that $S p$ analog of the discrete anomaly matching in the $S O(N)$ model does not generally work. In the $S p(2 N)$ theory, a $Z_{2 N-2}$ subgroup of the classical $U(1)_{A}$ group, which assigns the $A$ field charge 1 , remains intact at the quantum level. The $Z_{2 N-2}^{3}, Z_{2 N-2}^{2} U(1)_{R}$ and $Z_{2 N-2} U(1)_{R}^{2}$ anomalies, which need not match since they can be corrupted by the massive spectrum, indeed do not match. Furthermore, the difference between the microscopic and macroscopic values for the $Z_{2 N-2}$-gravity-gravity anomalies

$$
\begin{aligned}
& A_{Z_{2 N-2}}^{\text {(parton) }}=\left(2 N^{2}-N-1\right)(1)=(N-1)(2 N+1) \\
& A_{Z_{2 N-2}}^{\text {(hadron) }}=\sum_{n=2}^{N} n=\frac{1}{2}(N-1)(N+2)
\end{aligned}
$$

is $\Delta=\frac{3}{2} N(N-1)$. So the $Z_{2 N-2}$ anomalies match modulo $N-1$ if $N$ is even, but they fail to match if $N$ is odd.

Instances where naive discrete anomaly matching arguments fail have previously been noted [9]. For example, the nonvanishing vev for the baryonic glueball operator $B=$ $\epsilon_{\mu_{1} \cdots \mu_{N_{c}}} W^{\mu_{1} \mu_{2}} W^{\mu_{3} \mu_{4}} V^{\mu_{5}} \cdots V^{\mu_{N_{c}}}$ within $S O\left(N_{c}\right)$ theory with $N_{f}=N_{c}-4$ vectors leaves intact all continuous global symmetries but breaks the instanton induced $Z_{2 N_{c}-8}$ down to $Z_{N_{c}-4}$. Anomalies therefore match only for the latter discrete subgroup and not for its larger progenitor. A similar phenomenon may resolve the discrete anomaly mismatch in the symplectic model. O The $N$ glueballs which must emerge when $S p(2 N) \rightarrow S U(2)^{N}$ come from linear combinations of $B_{n}=\operatorname{Tr}(W J)^{2}(A J)^{n}$ where $0 \leq n \leq N-1$. The only one of these operators whose vev does not break $U(1)_{R}$ is $B_{N-1}$. If this glueball composite develops a nonzero expectation value, all anomalies involving the unbroken discrete group $Z_{N-1}$ match between the microscopic and macroscopic theories for $N$ even. Moreover, anomalies also match for $N$ odd provided the low energy sigma model contains an odd number of massive Majorana fermions with charge $N-1$. It is important to note that only an even number of such Majorana fermions can exist when $N$ is even if the prior

7 We thank Csaba Csàki for discussions on this point. 
anomaly results are not to be disrupted. This rather involved scenario appears to yield viable anomaly matching results within the symplectic model.

\section{Acknowledgments}

JB thanks Lance Dixon and Lisa Randall for discussions. PC thanks Per Kraus for collaborating at an early stage on this work and Philip Argyres, Howard Georgi, Martin Schmaltz, Nathan Seiberg, Matt Strassler and Sandip Trivedi for sharing their insights. KI thanks Nathan Seiberg for useful discussions and Gustavo Dotti and Aneesh Manohar for rekindling his interest in these models. We also acknowledge support from the Dept. of Energy under Grant DOE-FG02-91ER40671 (JB), the National Science Foundation under Grant \#PHY-9218167 (PC), and the Dept. of Energy under Grant DOE-FG03-97ER40506 and the Alfred Sloan Fellowship Foundation (KI). 


\section{References}

[1] For reviews, see K. Intriligator and N. Seiberg, hep-th/9509066, Nucl. Phys. Proc. Suppl. 45BC (1996) 1 ; M.E. Peskin, hep-th/9702094; M. Shifman, hep-th/9704114, Prog. Part. Nucl. Phys. 39 (1997) 1.

[2] For recent reviews of dynamical supersymmetry breaking, see W. Skiba, hepth/9703159; A. Nelson, hep-ph 9707442; E. Poppitz, hep-ph/9710274; S. Thomas, hep-th/9801007.

[3] K. Intriligator, N. Seiberg and S. Shenker, hep-th/9410203, Phys. Lett. B342 (1995) 152.

[4] G. Dotti and A.V. Manohar, hep-th/9712010.

[5] C. Csaki, M. Schmaltz, W. Skiba, and J. Terning, hep-th/9801207.

[6] J. Preskill, S. Trivedi, F. Wilczek and M. Wise, Nucl. Phys. B363 (1991) 207.

[7] L. Ibáñez and G. Ross, Phys. Lett. 260B (1991) 291; Nucl. Phys. B368 (1992) 3; L. Ibáñez, Nucl. Phys. B398 (1993) 301.

[8] T. Banks and M. Dine, Phys. Rev. D45 (1992) 1424.

[9] C. Csàki and H. Murayama, hep-th 9710105.

[10] G. 't Hooft, Phys. Rev. Lett. 37 (1976) 8; Phys. Rev. D14 (1976) 3432.

[11] K. Intriligator and N. Seiberg, hep-th/9503179, Nucl. Phys. B444 (1995) 125.

[12] K. Intriligator and N. Seiberg, hep-th/9506084, Proc. of Strings '95, edited by I. Bars et. al., (World Scientific, 1996)

[13] K. Intriligator, hep-th/9505051, Nucl. Phys. B448 (1995) 187.

[14] R.G. Leigh and M.J. Strassler, hep-th/9503121, Nucl. Phys. B447 (1995) 95.

[15] G. Parisi and N. Sourlas, Phys. Rev. Lett. 43 (1979) 744.

[16] R. Penrose, in Combinatorial Mathematics and its Applications, edited by D.J.A Welsh (Academic Press, New York, 1971).

[17] P. Cvitanovic, Group Theory, Nordita Notes (1984) 136.

[18] R.C. King, Can. J. Math. 23 (1971) 176.

[19] P. Cvitanovic and A.D. Kennedy, Phys. Scr. 26 (1982) 5.

[20] G.V. Dunne, J. Phys. A: Math. Gen. 22 (1989) 1719.

[21] P. Cho and P. Kraus, hep-th/9607200, Phys. Rev. D54 (1996) 7640.

[22] C. Csàki, M. Schmaltz and W. Skiba, hep-th/9607210, Nucl. Phys. B487 (1997) 128. 